\title{
Advanced Undifferentiated Endometrial Carcinoma with No Evidence of Disease after Neoadjuvant Chemotherapy: A Case Report
}

\author{
Cassandra L. Presti¹, Payam Katebi Kashi' ${ }^{1}$ Christopher M. Tarney², Ruchi Garg1,3 \\ ${ }^{1}$ Division of Gynecologic Oncology, Department of Obstetrics and Gynecology, Inova Fairfax Women's Hospital, Falls Church, \\ VA, USA \\ ${ }^{2}$ Walter Reed National Military Medical Center, Department of Obstetrics and Gynecology, Division of Gynecologic Oncology, \\ Bethesda, MD, USA \\ ${ }^{3}$ Mid Atlantic Gynecologic Oncology and Pelvic Surgery Associates, Fairfax, Virginia, USA \\ Email: cassandra.presti@inova.org
}

How to cite this paper: Presti, C.L., Kashi, P.K., Tarney, C.M. and Garg, R. (2020) Advanced Undifferentiated Endometrial Carcinoma with No Evidence of Disease after Neoadjuvant Chemotherapy: A Case Report. Open Journal of Obstetrics and Gynecology, 10, 1573-1579.

https://doi.org/10.4236/ojog.2020.10110141

Received: September 25, 2020 Accepted: November 13, 2020

Published: November 16, 2020

Copyright $\odot 2020$ by author(s) and Scientific Research Publishing Inc. This work is licensed under the Creative Commons Attribution International License (CC BY 4.0).

http://creativecommons.org/licenses/by/4.0/

\begin{abstract}
Background: Neoadjuvant chemotherapy is used in gynecologic malignancies as primary treatment for advanced disease and has demonstrated improvement in survival rates. However, the data for use in endometrial cancer is limited. Case: This is a case of stage IV endometrial undifferentiated carcinoma with complete response to four cycles of neoadjuvant chemotherapy (carboplatin/paclitaxel) and no evidence of disease during the interval debulking surgery (robotic-assisted total laparoscopic hysterectomy with bilateral salpingoophorectomy, pelvic and paraaortic lymph node dissection, and cystoscopy). Conclusion: Complete response to neoadjuvant chemotherapy prior to interval debulking surgery is only reported in a limited number of cases, and even less common in advanced stage high-grade endometrial cancers. The use of neoadjuvant chemotherapy may reduce tumor burden, increase opportunities for optimal debulking, and have an impact on survival. Further research is needed to understand the potential for neoadjuvant chemotherapy in these patients.
\end{abstract}

\section{Keywords}

Neoadjuvant Chemotherapy, Undifferentiated Endometrial Carcinoma

\section{Introduction}

Endometrial cancer is the most common gynecologic cancer in the United 
States. The increasing incidence and mortality from endometrial cancer persist despite advances in cancer recognition and treatment. The main treatment modality for advanced stage endometrial cancer is primary cytoreductive surgery. Additionally, patients who are poor surgical candidates or have unresectable disease have fewer surgical treatment options, often relying on radiation and chemotherapy.

The use of neoadjuvant chemotherapy (NACT) in epithelial ovarian cancer (EOC) is supported by multiple studies; however, data on the utility of such therapy in endometrial cancer is limited. European trials for advanced stage EOC have shown that the progression free survival (PFS) and overall survival (OS) in patients who had NACT followed by interval debulking surgery (IDS) are non-inferior to patients who underwent primary cytoreductive surgery [1]. Additionally, patients with advanced stage EOC who underwent NACT and IDS demonstrated lower rates of surgical mortality and higher optimal or complete debulking. Most of the current data that describes NACT in endometrial cancer is based on its use in EOC, as there is scarce data on advanced endometrial cancer subtypes such as endometrioid, clear cell, or carcinosarcoma [2].

Undifferentiated endometrial carcinoma is a rare subtype of endometrial cancer that often confers worse prognosis. There is insufficient evidence of using NACT with this histology. Here we report a case of a patient with stage IV undifferentiated endometrial carcinoma who underwent four cycles of NACT after her initial diagnostic laparoscopy and subsequently had a complete response with no signs of residual disease at the time of her IDS.

\section{Case Presentation}

The patient is a 63-year-old female who presented to her gynecologist with abdominal cramping and postmenopausal bleeding. She underwent an endometrial biopsy and was found to have adenosarcoma with sarcomatous overgrowth with foci of complex atypical hyperplasia. A computed topography (CT) of her chest, abdomen, and pelvis showed enlarged paraaortic and pelvic lymph nodes (Figure 1(a) and Figure 1(b)).

She was referred to a gynecologic oncologist for treatment of her endometrial cancer. The patient was consented for robotic-assisted total laparoscopic hysterectomy, bilateral salpingoopherectomy, lymph node dissection, and tumor debulking. Upon placement of urinary foley catheter, gross hematuria was observed. Laparoscopic evaluation of the abdominal cavity revealed evidence of tumor metastasis to the pelvic sidewall. The bladder was noted to be densely adherent and friable tumor was noted at the dome and mid-posterior bladder wall. Cystoscopy revealed small plaques concerning for tumor invasion into the bladder at the left dome and right trigone. No cystoscopic biopsies were performed due to concern for fistulization. The current disease in pelvis would have required an anterior pelvic exenteration and creation of permanent ostomies therefore the decision was made to abort current surgery. A dilation and curettage was performed for better endometrial tissue sampling. The patient had an 


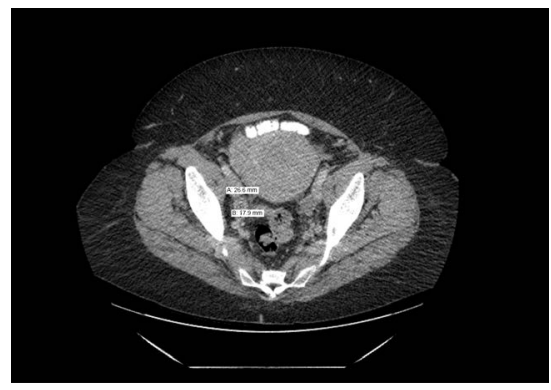

(a)

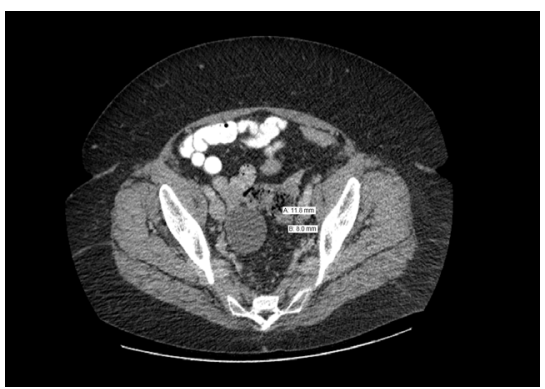

(c)

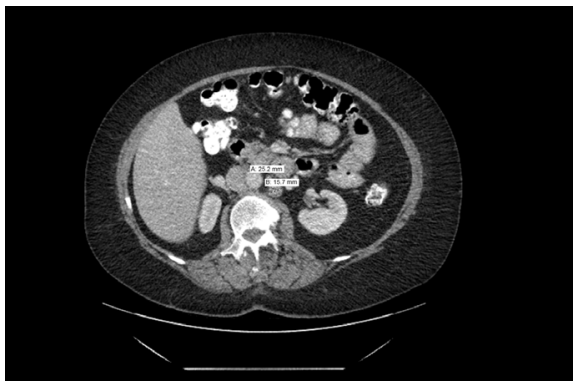

(b)

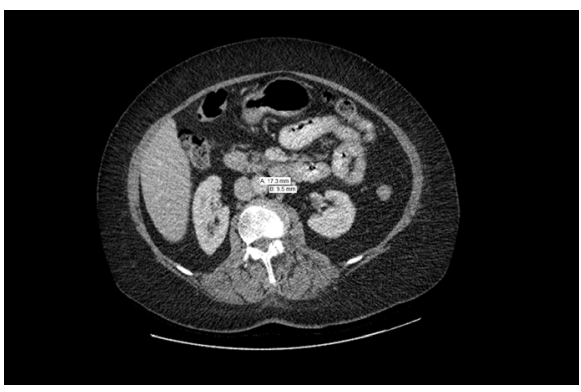

(d)

Figure 1. Pre- and post-chemotherapy CT of chest, abdomen, and pelvis. The CT revealed metastatic spread to aortocaval lymph nodes pre-treatment. Two large paraaortic nodes were identified measuring $2.6 \times 2.1 \mathrm{~cm}$ and $2.5 \times 1.6 \mathrm{~cm}$. Enlarged pelvic lymph nodes measured $2.2 \times 1.7 \mathrm{~cm}$ and $2.7 \times 1.8 \mathrm{~cm}$, on left and right iliac arteries respectively ((a) and (b)). The abdominal and pelvic nodes decreased at the post neoadjuvant chemotherapy imaging. Aortocaval node measured $1.6 \times 1.0 \mathrm{~cm}$ previously measured $2.5 \times 1.6$ $\mathrm{cm}$, left pelvic lymph node measured $1.2 \times 0.8 \mathrm{~cm}$ previously measured $2.2 \times 1.7 \mathrm{~cm}$ and right pelvic lymph node $1.1 \times 0.7 \mathrm{~cm}$ previously measured $2.7 \times 1.8 \mathrm{~cm}((\mathrm{c})$ and $(\mathrm{d}))$.

uncomplicated postoperative course and was discharged to home on postoperative day one. Pathology from the dilation and curettage revealed undifferentiated carcinoma in the endometrial curettage (EMC). The tissue stained positive for pancytokeratin, CK8/18, EMA, p16, and p53 (Figure 2). The tissue stained negative for PAX8, CD45, CD43, ER, PR, GATA3, and WT1. A pelvic MRI was done and no clear invasion was noted, however given the intraoperative findings, the patient was diagnosed with Stage IVA undifferentiated endometrial carcinoma.

She was treated with four cycles of NACT consisting of carboplatin (AUC 6) and paclitaxel $\left(175 \mathrm{mg} / \mathrm{m}^{2}\right)$ every 21 days. Subsequent CT scan showed interval improvement of lymphadenopathy and no new disease (Figure 1(c) and Figure $1(\mathrm{~d})$ ). She then underwent a robotic-assisted total laparoscopic hysterectomy with bilateral salpingoophorectomy, pelvic and paraaortic lymph node dissection, and cystoscopy. Operative findings included prominent bilateral obturator, bilateral external iliac, and right paraaortic lymph nodes, as well as bowel adhesions. No lesions were identified on cystoscopy. Final pathology showed no residual carcinoma in uterus/cervix/fallopian tubes with microscopic focus of atypical complex hyperplasia (Figure 3). Pelvic and paraaortic lymph nodes were also negative for malignancy. The patient had an uncomplicated postoperative 


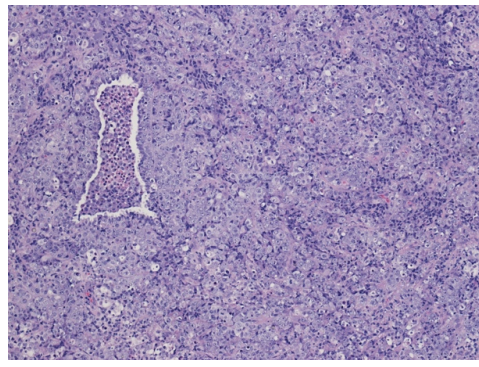

(a)

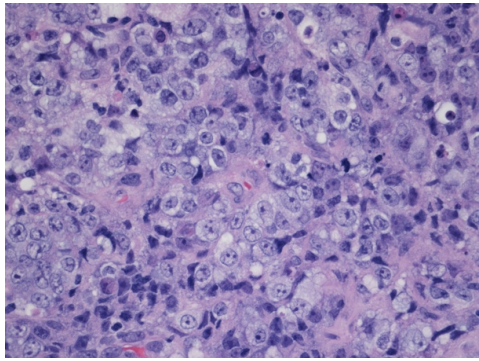

(c)

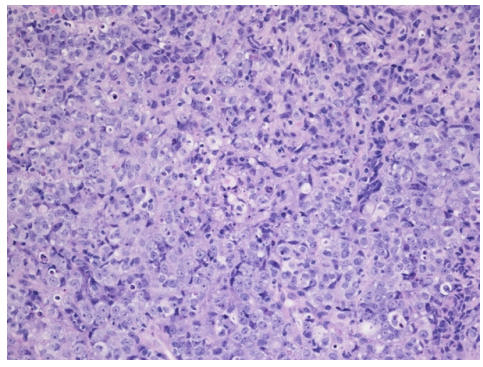

(b)

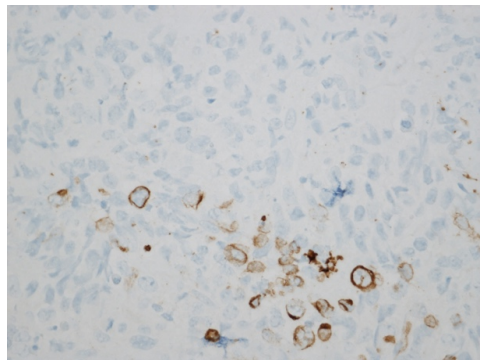

(d)

Figure 2. Endometrial tissue diagnosis. Endometrial tissue sampling, obtained by dilation and curettage. The undifferentiated carcinoma was found to have nests of large cells with little cytoplasm, enlarged vesicular nuclei, mitotic figures, and apoptotic bodies $(100 \times$, $200 \times$, and $400 \times)((a),(b)$, and (c) respectively). Mature lymphocytes are seen in the background (c). Cytokeratin AE1/AE3 immunostain shows the characteristic staining of rare tumor cells with lack of staining of majority of the tumor cells (d). Immunostains for PAX8, ER, PR, GATA3, and CD45 were all negative (not shown).

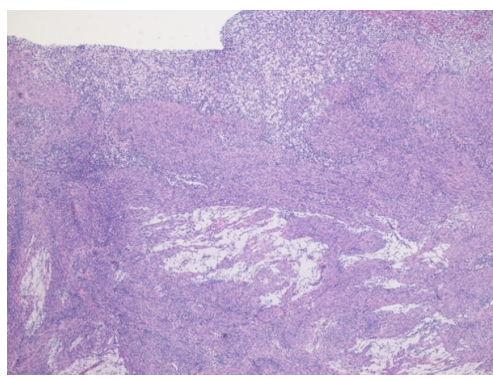

(a)

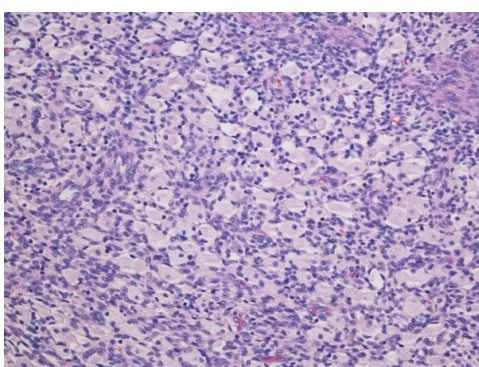

(b)

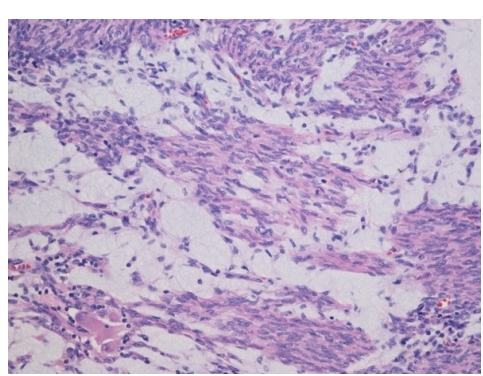

(c)

Figure 3. Uterine pathology after neoadjuvant chemotherapy. Areas of the uterine tissue demonstrated a histiocytic inflammatory reaction on the endometrial surface and within myometrial bundles $(100 \times, 200 \times$, and $400 \times)((a),(b)$, and (c) respectively). No residual tumor cells are seen. This is compatible with a complete treatment effect/response of the myoinvasive tumor. 
course. Four weeks after surgery, the patient received an additional two cycles of adjuvant carboplatin and paclitaxel. Upon review of the case at tumor board with radiation oncology, the decision was made to forego any radiation therapy. Currently, the patient is 44 weeks from IDS and has no signs/symptoms of recurrence.

\section{Discussion}

Surgery, chemotherapy, and radiation are all considered in the treatment regimen for endometrial cancer. Additionally, there are significant benefits in survival if optimal debulking can be achieved. Patients with advance endometrial cancer who had tumor less than $1 \mathrm{~cm}$ after debulking had a median survival of 15 months, while those with only microscopic disease had a median survival of 40 months [2]. Yet, the decision to manage advanced stage endometrial cancer with NACT and IDS or primary debulking surgery remains scarcely studied.

Previously, NACT has been used in the treatment of advanced gynecologic cancers, especially those patients who are deemed to be poor candidates for optimal primary debulking surgeries. The rate of optimal IDS has increased in endometrial cancer using NACT [3] [4]. There is evidence for NACT in stage III or greater endometrial cancer patients-those with positive lymph nodes [5]. Other studies suggest that the combination of NACT and IDS should be considered regardless of histologic subtype [6]. Patients that have undergone NACT followed by IDS have had a high rate of optimal cytoreduction and as a result, improved OS [7]. Although upfront NACT rarely diminished advanced endometrial cancer completely, the combination of NACT and IDS had positive impact on both PFS and OS [6]. Nevertheless, a recent study showed that less than half of endometrial cancer patients who received NACT underwent IDS [7].

In this case, NACT was utilized for primary therapy of advance stage endometrial cancer. After initial diagnostic surgery revealed stage IV endometrial cancer, this patient was treated with the recommended NACT, before proceeding with IDS. However, this case demonstrates a unique outcome of no evidence of disease (NED) after only four cycles of NACT in an undifferentiated carcinoma. The combination of carboplatin and paclitaxel is commonly used for treatment of endometrial cancer. This patient's complete response to carboplatin/paclitaxel highlights the potential benefits of NACT with IDS, as described above.

There are only a few reports of complete response to NACT in endometrial cancer. A study of 39 patients with endometrial cancer (10\% of which were sarcomas) from 2002-2016 showed only 56\% partial response and no complete response to NACT [7]. Two prior cases of advanced serous endometrial carcinoma were described as having no residual tumor after NACT [8]. To our knowledge, no prior cases of complete response in undifferentiated endometrial carcinoma after four cycles have been reported. As complete responses to NACT are rare, it is important for future studies to explore the patient populations or tumor ge- 
netics that are most likely to become NED after NACT. This approach allows patients with specific endometrial cancer subtypes to benefit from optimal IDS without undergoing radical resection and possible temporary or permanent urinary or bowel diversion procedures.

In conclusion, complete response to NACT prior to IDS is only reported in a limited number of cases. This case demonstrates one such case after four cycles of NACT with carboplatin/paclitaxel. Additional research is needed to make recommendations about the use of NACT with IDS when compared with primary debulking surgeries in advanced stage endometrial cancer.

\section{Acknowledgements}

We would like to thank Saeid Movahedi-Lankarani MD for his assistance and contributions to the pathologic evaluation and descriptions in this case report.

\section{Disclosures}

Dr. Ruchi Garg is a speaker for AstraZeneca. The other authors (Presti CL, Katebi Kashi P, and Tarney $\mathrm{CM}$ ) have no affiliations with or involvement in any organization or entity with any financial interest (such as honoraria; educational grants; participation in speakers' bureaus; membership, employment, consultancies, stock ownership, or other equity interest; and expert testimony or patent-licensing arrangements), or non-financial interest (such as personal or professional relationships, affiliations, knowledge or beliefs) in the subject matter or materials discussed in this manuscript. This study received no financial support. The opinions or assertions contained herein are the private views of the authors and are not to be construed as the official policy of Inova Fairfax Hospital, the Department of the Army, Navy, Air Force, Department of Defense, or the U.S. Government.

\section{Conflicts of Interest}

The authors declare no conflicts of interest regarding the publication of this paper.

\section{References}

[1] Vergote, I., De Wever, I., Tjalma, W., et al. (1998) Neoadjuvant Chemotherapy or Primary Debulking Surgery in Advanced Ovarian Carcinoma: A Retrospective Analysis of 285 Patients. Gynecologic Oncology, 71, 431-436. https://doi.org/10.1006/gyno.1998.5213

[2] Burke, W.M., Orr, J., Leitao, M., et al. (2014) Endometrial Cancer: A Review and Current Management Strategies: Part I. Gynecologic Oncology, 134, 385-392. https://doi.org/10.1016/j.ygyno.2014.05.018

[3] Vandenput, I., Van Calster, B., Capoen, A., Leunen, K., Berteloot, P., Neven, P., Moerman, P., Vergote, I. and Amant, F. (2009) Neoadjuvant Chemotherapy Followed by Interval Debulking Surgery in Patients with Serous Endometrial Cancer with Transperitoneal Spread (Stage IV): A New Preferred Treatment? British Jour- 
nal of Cancer, 101, 244-249. https://doi.org/10.1038/sj.bjc.6605157

[4] Takami, M., Ohta, Y., Nakayama, Y., Fukai, H., Matsumoto, H., Takimoto, T., Sakamoto, H. and Yamam (2007) A Case of Advanced Clear Cell Carcinoma of the Endometrium That Responded Remarkably to Neoadjuvant Chemotherapy of Combination Carboplatin Plus Weekly Paclitaxel. Gan To Kagaku Ryoho, 34, 457-460.

[5] Bestvina, C.M. and Fleming, G.F. (2016) Chemotherapy for Endometrial Cancer in Adjuvant and Advanced Disease Settings. The Oncologist, 21, 1250-1259. https://doi.org/10.1634/theoncologist.2016-0062

[6] de Lange, N.M., Ezendam, N.P.M., Kwon, J.S., Vandenput, I., Mirchandani, D., Amant, F., Van der Putten, L.J.M. and Pijnenborg, J.M.A. (2019) Neoadjuvant Chemotherapy Followed by Surgery for Advanced-Stage Endometrial Cancer. Current Oncology, 26, e226-e232. https://doi.org/10.3747/co.26.4655

[7] Khouri, O.R., Frey, M.K., Musa, F., Muggia, F., Lee, J., Boyd, L., Curti, J.P. and Pothuri, B. (2019) Neoadjuvant Chemotherapy in Patients with Advanced Endometrial Cancer. Cancer Chemotherapy and Pharmacology, 84, 281-285. https://doi.org/10.1007/s00280-019-03838-x

[8] Despierre, E., Moerman, P., Vergote, I. and Amant, F. (2006) Is There a Role for Neoadjuvant Chemotherapy in the Treatment of Stage IV Serous Endometrial Carcinoma? International Journal of Gynecological Cancer, 16, 273-277. 